Escuela de Ciencias Sociales y Humanidades, UNED, C.R.

URL: http://investiga.uned.ac.cr/revistas/index.php/espiga/index

ISSN: 1409-4002 • e-ISSN: 2215-454X

doi: http://dx.doi.org/10.22458/re.v17i34.1596

\title{
Entre el no saber del que enseña y el no saber del que aprende: una reflexión desde la práctica filosófica
}

\author{
Diego Pereira-Ríos* \\ iD https://orcid.org/0000-0002-2202-915X
}

Recibido: 24 de noviembre, 2016 • Aceptado: 26 de abril, 2017

\section{RESUMEN}

El presente trabajo intenta dar cuenta de lo que ha significado la filosofía para el autor en su camino por la docencia, y sus percepciones a lo largo de los años de formación profesional. El proceso educativo incluye un aprendizaje teórico y práctico que implica el manejo de ciertos saberes y una puesta en práctica de estos. El autor reflexiona acerca de la misma filosofía y su didáctica, con el fin de dar cuenta de que la filosofía implica una didáctica propia, en la cual se descubren dificultades planteadas en el curso con los alumnos de la enseñanza secundaria, cuando la dinámica del aula nos revela una complejidad que enriquece el mismo pensar filosófico. La cuota de responsabilidad intelectual como docentes pone en tela de juicio la vocación filosófica de quien asume el desafío, entre otros aspectos tratados. Por eso, si bien se presenta una reflexión desde la experiencia del mismo autor como docente, el artículo puede ser un insumo válido para seguir pensando en la necesidad de una didáctica filosófica.

Palabras clave: filosofía, didáctica, saber teórico, práctica, sabiduría.

\section{Formato de citación según APA}

Pereira-Ríos, D. (2017). Entre el no saber del que enseña y el no saber del que aprende: Una reflexión desde la práctica filosófica. Revista Espiga, 16(34), 255-269. Doi: http://dx.doi.org/10.22458/re.v17i34.1596

\section{Formato de citación según Chicago-Deusto}

Pereira-Ríos, Diego. «Entre el no saber del que enseña y el no saber del que aprende: una reflexión desde la práctica filosófica». Revista Espiga 16, n. ${ }^{\circ} 34$ (julio-diciembre, 2017): 255-269. Doi: http://dx.doi.org/10.22458/re.v17i34.1596

* Profesor de Filosofía (culminando). Bachillerato en Filosofía y primer año en la licenciatura en Ciencias Teológicas en la Facultad de Teología Monseñor Mariano Soler. Universidad de Montevideo. Uruguay. Correo: pereira.arje@gmail.com 


\section{Introducción}

En Uruguay existe un buen camino recorrido en la formación profesional de docentes para el ejercicio de la enseñanza en el nivel medio. Hablamos de sesenta años de una continua revisión y modificación de los métodos, planes y fundamentos acerca de cómo ir mejorando y perfeccionando la relación educativa entre docente y alumno. Sobre todo se fue percibiendo la necesidad de una especificidad de las diversas asignaturas.

En referencia a la Filosofía se comienza a trabajar con los alumnos entre los 15-18 años, en los últimos tres años del bachillerato, para lo cual los futuros docentes deben atravesar como mínimo cuatro años de formación académica que es a su vez teórica y práctica. La didáctica filosófica intenta dotar al alumno-docente de conocimientos académicos (pedagógicos, disciplinares, didácticos y normativos), pero también de impulsar un compromiso intelectual y práctico que lo lleve a acompañar a sus alumnos en el proceso de aprendizaje. Se busca alcanzar la toma de conciencia de que «...El profesor de filosofía es un factor esencial en el proceso educativo» «....apuntamos hacia las cualidades y virtudes que éste debe poseer cuando enseña Filosofía...» ${ }^{1}$.

Durante los cuatro años de formación el alumno-profesor atraviesa un primer año donde recibe una «introducción a la didáctica de la filosofía», mientras el resto de los tres años incluyen las clases teóricas con el docente de didáctica, y su puesta en práctica en alguno de los institutos de enseñanza secundaria asignados, donde lo acompaña un profesor adscriptor. En el camino se ayuda al futuro docente a realizar un autoconocimiento para el ejercicio de la docencia, pero también una reflexión crítica acerca de la educación y de la enseñanza de la Filosofía. Se trata que el alumno se inicie en la problematización de la misma enseñanza de la Filosofía haciendo de ello un problema filosófico. A diferencia de la didáctica clásica, como aprendizaje de teorías y de sistemas filosóficos con fines formativos, como disciplina necesita «...una didáctica exigida por la naturaleza particular de la filosofía, implicará una filosofía de la educación en el acto mismo de enseñar y de aprender, es decir, en la dimensión educativa que ese acto debe tener, por la especificidad de lo que se enseña y es aprendido...»².

Por tanto, el trabajo es una reflexión de lo que han sido estos años de formación en la docencia en conjunción con la experiencia de la práctica con los alumnos. Pensar es siempre pensar-con-otros, desde otros y hacia otros. Como parte de los ejes centrales de este trabajo tomaré ideas del profesor uruguayo Mauricio Langón y su artículo: «Filosofía de la enseñanza de la Filosofía ${ }^{3}$, y también del filósofo francés Françoise Raffin y su artículo "Nuestra concepción de la didáctica» ${ }^{4}$ para intentar esbozar algunas ideas acerca de la didáctica de la filosofía y, a su vez, lo descubierto en la misma práctica del profesorado. Teoría y práctica, ideas y experiencia se irán mezclando en los siguientes renglones

1. Augusto Salazar Bondi, Didáctica de la filosofía (Lima: Fondo Editorial, 1995), 47.

2. João Boavida, «De una didáctica de la filosofía a una filosofía de la educación», Revista Española de Pedagogía 64, n. ${ }^{\circ} 234$ (mayo-agosto, 2006), 205-225.

3. Mauricio Langón, «Filosofía de la enseñanza de la filosofía» (Comunicación al Congreso Brasileiro de Professores de Filosofía. Piracicaba, noviembre 2000).

4. Francoise Raffin, «Nuestra concepción de la didáctica» (Traducción Langón, M.), Revista Diotime L'Agora (2000). 
buscando nuevas pistas, algunas señales, pequeños indicios del andar propio de la filosofía. La ausencia de respuestas se convierte en el deseo y con él surgen las preguntas que buscan hacer presente lo ausente. Las respuestas pueden aparecer al intentar formular buenas preguntas. He aquí el desafío de todo filósofo.

\section{Breve impresión de los textos seleccionados y planteamiento del trabajo}

Al leer el texto de Langón se logra experimentar «eso» que nos sucede cuando se lee un texto sencillo de comprender, pero que esconde detrás de cada palabra una carga inmensa de sabiduría fruto del estudio, pero también de la experiencia vivida, y de un gran sentido común. En el texto además de nutrir al lector de un gran bagaje conceptual, se percibe que nunca olvida la realidad del aula y de la experiencia del trabajo filosófico con los alumnos. Sería lo que Deleuze y Guattari dicen del filósofo al describirlo como amigo del concepto ${ }^{5}$, ya que al leerlo el vocabulario del lector no sólo experimenta que se enriquece, sino que también siente la necesidad de seguir leyendo más pues el autor logra motivarlo. Pero sería muy injusto afirmar que el texto referido sólo es rico en conceptos: la lectura de Langón logra que quien lea con preocupación filosófica se cuestione, reflexione acerca del llamado o la invitación (in-vocación) propia a la Filosofía. La preocupación por aprender-enseñar a filosofar se va transformando en una preocupación por el ser humano.

El otro texto elegido para el trabajo tiene la intención de buscar una base sólida a la hora de lograr cierta independencia como profesional de la educación, algo tan necesario para todo docente. Raffin aporta ideas claras de cómo «bajar a la clase» las ideas, búsquedas, intensiones, alcances, de un curso de filosofía. Todo ello en el marco del profesor como autor de su curso, como quien debe organizar el trabajo para alcanzar algunas metas que se proponga en relación con el grupo concreto con el cual esté trabajando. También insiste en la didáctica de la filosofía como actividad compleja debido a su especificidad, dándonos algunos indicios que hacen que no la tomemos tan rápido, sino que exige un encare serio y detenido, y una reflexión profunda y sistemática.

Lo que intento a continuación es conjugar la lectura de los textos citados, con su respectivo análisis, pero sumándole algo de mi reflexión personal, siempre mirando hacia la práctica desarrollada a lo largo de estos cuatro años de formación en el profesorado. El texto se organiza en pequeños bloques temáticos que parten de aquellas inquietudes que han surgido en la lectura con aportes de otras lecturas seleccionadas, que ayudan e impulsan a escribir. Pero no hay que perder de vista lo central: es un camino de reflexión-acción-reflexión que implica no sólo la propuesta de ideas o la descripción de estrategias teórico-prácticas, sino que son reflexiones desde la acción realizada y vuelta a reflexionar. Esa es la gran riqueza de una verdadera didáctica filosófica, pues es un círculo que nunca se cierra sino que un primer principio va en busca de un supuesto punto de encuentro para luego volver a empezar. Esta es la grandeza de la filosofía, como dijo Beltrand Russell: «De hecho el valor de la filosofía debe ser buscada en una larga medida en su real incertidumbre.... ${ }^{6}$.

5. Cfr. Gilles, Deleuze y Félix Guattari, ¿Qué es la filosofía? (Barcelona: Anagrama, 2005), 11

6. Beltrand Russel, Los problemas de la filosofia. Acceso: 12 febrero, 2018, http://www.enxarxa.com/biblioteca/RUSSELL $\% 20$ Los $\% 20$ problemas $\% 20$ de $\% 201$ \% $\% 20$ filosofia.pdf, 76. 


\section{Primero: la filosofía como búsqueda de sabiduría}

Embarcarnos en un camino de enseñanza de la filosofía tiene una meta que puede provocarnos temor: la búsqueda de una cierta sabiduría, que nos atrae hacia sí de una forma muy particular. Es particular pues podemos creer que mediante el estudio y la reflexión crítica podemos alcanzar cierto nivel de sabiduría. Pero la misma vida nos va mostrando las limitaciones que tenemos como docentes. Por otro lado, en ocasiones la misma sabiduría nos provoca de tal manera que nos hace buscarla por todos los medios posibles. Si sabiduría tiene que ver no sólo con poseer cierta capacidad intelectual sino sobre todo de saber encontrarle sabor a las cosas, en filosofía tiene que ver con saber gustar de las cosas que la vida nos provee (situaciones, dificultades, oportunidades, sorpresas) para darles un sentido importante para cada uno. Ello nos lleva a involucrarnos cada vez en las cuestiones existenciales de nuestro tiempo, pues nuestros alumnos son hijos de este tiempo, y necesitan desarrollar la capacidad de darle sentido a todo lo que experimentan, impulsándolos no solo por el gusto de hacerlo, sino por la necesidad de aprender a pensar.

Y al pensar la filosofía lo hago como el desafío de adentrarme en la búsqueda de esa sabiduría tan íntima y personal -pero a su vez tan comunitaria- de aprender a vivir, o de lograr caminos para vivir bien con el resto de la sociedad. Se trata de encontrar soluciones a los problemas más importantes de la existencia y que pueden hacer del mundo un lugar mejor. Por eso la sabiduría que debiéramos buscar en las clases de filosofía andaría por estos caminos de lograr que nuestros alumnos utilicen mejor sus facultades naturales (intelectuales, artísticas, sentimentales, prácticas, teóricas, imaginativas) para que logren orientar su vida con una mayor criticidad intentando una coherencia entre su pensar y su actuar. Langón expresa esto de manera excepcional: «...no se trata, en educación, de aprender lo ya sabido, sino de aprender a ser humano a través de las actividades educativas...» ${ }^{7}$.

Este aprender a ser humano no sólo implica un gran esfuerzo intelectual de desarrollar una necesaria capacidad crítica con la cual mirar la realidad, sino que también una gran apertura a lo que mueve a nuestros alumnos: la música, sus gustos, sus miedos, sus esperanzas, sus alegrías, sus sufrimientos, sus pasatiempos, sus búsquedas, sus expectativas, etc. Sería intentar encauzar las energías vitales de los adolescentes y jóvenes -que tiende a alcanzar la felicidad- y atraerla hacia la actividad filosófica, buscando sensibilizar desde la misma filosofía. Dice Langón en otro artículo: «...en la adolescencia, filosofar se hace cosa de vida o muerte, de sentido de la vida, de inserción en el complejo proceso de cam-

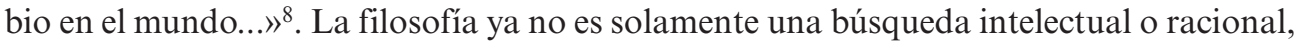
sino que se convierte en una búsqueda de sabiduría vital, de caminos de realización, de razones imprescindibles que muevan todas las dimensiones del ser humano.

7. Mauricio Langón, «Adolescencias de la filosofía» (Comunicación a las I Jornadas Internacionales de Filosofía, Infancias en la filosofía: experimentar el pensar, pensar la experiencia, Buenos Aires, Novedades Educativas, 22-23 de julio de 2005).

8. Langón, «Adolescencias de la....». 


\section{Segundo: la filosofía como búsqueda de amor}

La cuestión aquí es buscar amar, pero ¿a quién? o ¿a qué? El concepto mismo de filosofía nos remite a pensar en amor a la sabiduría. Este amor propongo pensarlo en dos sentidos: por un lado el amor del docente por la filosofía, su pasión por el saber, el amor a su misma vocación, y la pasión por enseñar; y por otro lado el amor en la relación docente-alumno, en la necesaria interrelación del acto pedagógico, en su verdadera preocupación por la educación de sus alumnos. Pero es esta una relación de reciprocidad, como propone Puchet en referencia a Sócrates y Platón: «...hay una centralidad del cuidado de sí en la relación pedagógica establecida entre maestro y discípulo en el contexto socrático-platónico. El deseo nos lleva a buscar lo que verdaderamente hay que amar, que es la sabiduría.... $\rangle^{9}$. Veamos estos elementos detenidamente.

El primero tiene que ver con que en su ser docente de filosofía cada sujeto debe desarrollar su ser filósofo y provocar existencialmente el pensar. Es una cuestión de querer vivir, una provocación vital. No debe haber obligación sino, una necesidad imperante de ponerse a pensar. Este pensar atrae y cuestiona, impulsa a investigar lo que vivimos, pero también lo que no sabemos y que necesitamos saber. Por ello la vocación a la docencia de filosofía tiene influencia directa en lo que será luego la práctica concreta. Langón cita a Kant afirmando que no se puede aprender filosofía sin aprender a filosofar y creo que esto es una gran verdad. Ser docente de filosofía implica amar a la filosofía y darle un lugar de importancia dentro de la agenda cotidiana que poco a poco puede ser transmitida a los alumnos desde el ejemplo.

Por otro lado, pensar una vocación a la filosofía, sin un ejercicio responsable de filosofar, creo que nos puede inducir a muchos errores. Debe haber un apasionamiento por enseñar, pero sobre todo por seguir aprendiendo desde la lectura y el trabajo con otros, pasando incansablemente por la realidad propia y ajena, para lograr profundizar en la comprensión de la complejidad humana, ya que es allí donde descubrimos esa necesidad de saber. Educar es, básicamente, mover a filosofar, dice Langón y esto habla de la necesidad de conmoverse, de provocar un movimiento en el pensamiento propio y el de aquellos que escuchan. En la vocación del docente de filosofía debe haber una especie de responsabilidad de mover a pensar desde sí mismo, con los otros, y que los otros piensen entre sí.

El segundo sentido tiene, en relación con los alumnos -algo que pareciera simple consecuencia de lo anterior-, es el amor que debe brotar en el docente por sus alumnos. El maestro es maestro para sus alumnos y por ello debe existir un amor que surja de la esencia misma del acto de enseñar. Octavi Fullat propone seguir la enseñanza de Aristóteles en la Metafísica, explicando que la educación como factum, es un hecho donde el maestro domina la empeiría, o sea, posee la experiencia para saber actuar en función del bien de su alumno: «...a veces el educador actúa como tal porque sabe que aquel método, por ejemplo, resulta eficaz. Este es el nivel del curandero...» ${ }^{10}$. Amar a los alumnos sería entonces desear, querer, buscar que logren desarrollar la capacidad de pensar, decir, decidir y actuar, pero sin que sus alumnos pierdan su subjetividad, su mismidad. Se

9. Genis, Andrea, en Enrique Puchet, «De filosofía y educación. Cuidado de sí y conocimiento de sí» (Montevideo: De la Fuga, 2013), Epílogo, 82.

10. Octavi Fullat, El pasmo de ser hombre (Barcelona: Ariel, 1994), 51. 
trata de enseñar reparando lo que está mal, pero dando paso a la libertad. Esto normalmente es difícil, ya que durante los años de estudio hay mucha dependencia intelectual, pero por eso no hay que dejar pasiva la misma actividad del sujeto que busca aprender, y desde ello se hace necesario el amor al sujeto y el deseo de que sea realmente libre haciéndose cargo de sí: «El maestro de la inquietud de sí, insta a que el discípulo tenga inquietud de sí, se preocupe por sí mismo, entre en un proceso de autoconocimiento que transforme su vida $»^{11}$.

\section{Tercero: la filosofía necesita de maestros}

Dije anteriormente que el maestro es maestro para sus alumnos, pero no porque él lo decide, sino porque es elegido por ellos. Nosotros mismos, que pasamos de ser estudiantes en el profesorado, en vistas de ser profesores, no seguimos de la misma manera a todos los docentes de nuestros centros de formación. Tendremos más empatía con unos que con otros, pero es real que necesitamos de maestros. En este momento surge la necesidad de cuestionarnos a nosotros mismos como maestros, algo que puede provocar cierto temor o vergüenza pero que en la realidad de nuestras clases de práctica es en lo que nos vamos configurando. De nuevo Fullat propone otro aspecto del maestro como educador: «...En otras ocasiones el educador interviene sobre el comportamiento del educando con un hacer que responde al conocimiento del por qué del éxito. Es cuestión de la tekhne...» $\rangle^{12}$. Aquí el docente debe ser consciente del hacer propio y del alumno, pero llevando su saber-hacer para todos, lo que implica una relación de cercanía con sus alumnos, de conocimiento mutuo.

Si el paradigma de maestro sigue siendo Sócrates, que filosofando hacía filosofar ${ }^{13}$, nos parece que siempre estaremos muy lejos de ello, pero no puede dejar de estar dentro del ideal al cual debemos aspirar. Para ir caminando hacia ello podemos cuestionarnos acerca de nuestras clases de filosofía y cuál es nuestra actitud al estar en el rol de maestro. Es interesante ver cómo Langón propone pensar sobre la compasión, como la capacidad de apasionarse con los demás, de apasionarse juntos. Para ello veo necesario la atención que debemos tener por nuestros alumnos para poder compartir con ellos lo que les apasiona -como dije antes- para atraerlo al trabajo filosófico.

Conocer a nuestros alumnos, interesarse por sus cosas, preocuparse por ellos, es parte del sentir-con que no sólo refiere a experimentar simpáticamente sus sentimientos; es también con-sentir con el otro en lo que piensa, percibe, cree acerca de la vida, y desde ese compartir traerlo al aula para reflexionar. Al hablar de con-sentir lo expreso como el intento de comprender al otro en su experiencia, en sus ideas y sentimientos, generando un espacio de confianza para que se exprese -ya que sus vivencias son verdaderas para él-, pero sin dejar de ayudarlo a cuestionarse, desde la experiencia de los demás alumnos y la propia experiencia del docente. De aquí la importancia de que el profesor logre armar un curso -y sentirse su autor- con base en sus alumnos: contenidos, necesidades, competencias, tiempos, estrategias, etc. Raffin dice: «...cada curso es, por ello, una puesta

11. Genis, Andrea, en Enrique Puchet, «De filosofía y...», 82.

12. Fullat, El pasmo de..., 52 .

13. Langón, «Filosofía de la enseñanza de la...» 
en obra singular...» donde «...un curso tiene siempre algo de acontecimiento...» ${ }^{14}$. Y si bien el acontecimiento tiene ese algo de novedoso, el terreno se puede preparar mejor si se conoce a los alumnos.

Al reclamar esta necesidad de un maestro como connatural a la filosofía, me baso en las dificultades que se generan ante tanta información que provoca la tergiversación de la esencia misma de la filosofía. Sobre todo cuando hoy la filosofía sigue sin ser valorada en su real aporte a la humanidad y siendo percibida como una disciplina sin sentido. También está la percepción de que la filosofía no tiene ninguna finalidad exterior, ya que no es útil, no enseña una pragmática, no es productiva o eficaz ${ }^{15}$. $\mathrm{Al}$ correr el peligro de ser incomprendida es necesario maestros que ayuden a los alumnos comprender lo incomprensible «...de esta disciplina de lo indisciplinable, para esta enseñanza de lo inenseñable, para este saber que es no-saber y más que saber...» ${ }^{16}$. Si bien el cultivo personal es una exigencia, se hace necesaria la presencia del maestro como guía, como espacio de consulta a quién acudir.

\section{Cuarto: la filosofía como búsqueda de expresión de nuestro pensar}

En este punto me interesa reflexionar acerca de la importancia de la filosofía desde nuestra tierra latinoamericana para intentar repensar los problemas que podemos trabajar. Quizá desde hace tiempo tengamos claro la necesidad de ahondar en el pensamiento latinoamericano, pero no siempre lo podemos percibir en los programas de formación docente. Si bien es necesaria la historia de la filosofía universal, también es necesaria una profundización en el pensamiento y reflexión que ya ha alcanzado la filosofía latinoamericana. Pensar desde este marco de referencia quizá nos ayude a repensar nuestros cursos de filosofía buscando situarnos en una realidad que tenga más que ver con nosotros y nuestros alumnos.

Partiendo de América Latina la filosofía tiene una gran diferencia histórica respecto a la filosofía europea de siglos anteriores en la cual nos seguimos formando. La compasión de la que habla Langón tiene en nuestro contexto significativas diferencias a la hora de pensar filosóficamente. La experiencia de sufrimiento, de opresión y de explotación, de lo que ha desaparecido de nosotros, de nuestras raíces, de nuestras costumbres, nos debe hacer pensar una filosofía que busque de alguna manera la reivindicación de nuestro pensar-sentir-hacer en lo que somos y deseamos ser. Se hace necesaria una filosofía desde espacios de liberación del pensamiento, de reflexión genuina desde nuestro propio ser latinoamericano. Dice Kusch: «Lo americano no es una cosa. Es simplemente consecuencia de una profunda decisión por lo americano entendido como un despiadado aquí y ahora y, por ende, como un enfrentamiento consigo mismo» ${ }^{17}$. Desde esto la propuesta de un curso de filosofía es ante todo una decisión de querernos y valorarnos dentro del mundo.

14. Raffin, «Nuestra concepción de la...»

15. Cfr. Jacques Derrida, «Las antinomias de la disciplina filosófica» (Carta enviada a los participantes del Colloque Rencontres Ėcole et Philosophie, realizado en la Universidad de París X, Nanterre, 20 y 21 de octubre de 1984).

16. Ibídem.

17. Rodolfo Kusch, Geocultura del hombre americano (Córdoba: Fundación Ross, 1976), 105. 
No se intenta con esto el rechazo a la influencia del pensar europeo sino que una nueva comprensión desde una cosmovisión diferente. Debemos aprovechar los grandes aportes a la filosofía de todos los pensadores europeos que nos siguen enriqueciendo, pero con la debida contextualización. Es lo que refiere Sambarino al hablar de una filosofía auténticamente latinoamericana: «América Latina está en un tiempo particularmente crítico, que invita a un pensar radical no necesitado de la mediación del pensar extranjero, sin perjuicio de usarlo en lo que selectivamente importe y sea eficaz. Es más, ya tiene y desde hace tiempo, un pensar propio y una conciencia de poseer una problemática particular» ${ }^{18}$. Si lográramos hoy nombrar algunas de estas problemáticas de la cual habla Sambarino, podríamos repensar nuestros cursos de filosofía centrados en problemas que logren conmover más aun a nuestros alumnos, y que los lleve a involucrarse con mayor responsabilidad.

El trabajo que viene realizando Fornet-Betancourt en este sentido es muy rico. El contexto global -y el latinoamericano específicamente- es de un alto grado de interculturalidad y por ello la filosofía debe tener en cuenta este elemento. Junto con él: «queremos una filosofía mejor; una filosofía más "colorida", pluricromática, pluriforme y plurivisional; pero si queremos operar en ella esta transformación es para que esté mejor preparada y equipada para cumplir su función como factor de cambio en nuestro presente histórico» ${ }^{19}$. Y esto se conjuga con la propuesta de Raffin al proponer como punto de partida de un curso de clase, la experiencia de los alumnos, para convertir los problemas en preguntas como ayuda al alumno a clarificar sus ideas.

Al preguntarme si es posible conjugar la historia latinoamericana y las necesidades vitales de los alumnos me convenzo de que sí, es posible desde la actualidad, ya que las políticas económicas dominantes, ayudados por los medios de comunicación, siguen haciendo que nuestra mirada se desvíe hacia Estados Unidos y Europa como los modelos de seres humanos a seguir. Entonces podemos decir que «...la experiencia del alumno es material indispensable que el profesor debe conocer y utilizar en la clase de filosofía... $\rangle^{20}$ y con ello poder armar el curso centrado en problemas que atraigan la atención de los alumnos buscando un mayor interés. Pueden ser los clásicos problemas de la filosofía (libertad, verdad, Dios, etc.) o nuevos problemas que tomen en cuenta el contexto intercultural en donde vivimos, o tomar los problemas y resignificarlos. Dice Sambarino: «un problema filosófico es auténtico cuando se encuentra situado en relación con la problemática radical de una configuración histórico cultural» ${ }^{21}$ ¿Podríamos problematizar sobre liberación en vez de libertad? ¿Sobre el sufrimiento antes que el amor? ¿Sobre la explotación antes que el bien superior? Quizá sea tarea de los filósofos de la educación repensar éstos problemas.

18. Mario Sambarino, «La función sociocultural de la filosofía en América Latina». Primer Coloquio Nacional de Filosofía de México: Morelia-Michocán, 1975, en La Filosofía actual en América Latina. Grijalbo, México (1976), 179.

19. Raúl Fournet-Betacourt, Transformación intercultural de la filosofía (Bilbao: Desclée de Brouwer, 2001), 174.

20. Raffin, «Nuestra concepción de la...».

21. Sambarino, «La función sociocultural...», 172. 


\section{Quinto: la filosofía como obra en construcción}

La filosofía tiene la capacidad de no imponer ni exigir un solo estilo de enseñanza o de aprendizaje. Nos da una gran libertad de acción, de propuestas metodológicas, incluso de intercambio de estrategias con otras disciplinas. Partir de la experiencia del alumno o partir de un texto filosófico, atentos a lo que provoca en ellos, pueden ser escusas con el fin de problematizar la realidad en la cual vivimos. Por ello Raffin afirma: «...es en este sentido que se dice a menudo que el profesor es el autor de su propio curso.... $\rangle^{22}$. Pero esta afirmación debemos tomarla en serio ya que a menudo se banaliza. Dice Fullat: «...una educación es un sistema de intervenciones mediante actos sobre propiedades, situaciones y procesos humanos con ánimo a obtener modificaciones en ellos...el acto educativo es enérqueia de una dynamis, enérqueia que presenta dos aspectos: la póiesis o producción y una praxis o acción... $\rangle^{23}$. Toda preparación previa del material a trabajar implica una consecuente práctica que debe luego cuestionar lo armado.

Pensarse como autor del curso que vamos a proponer a nuestros grupos de clase nos exige primero, como ya lo hemos dicho, un conocimiento de los alumnos, de sus experiencias de vida, que nos ayudan a jerarquizar los contenidos con base en las inquietudes, interrogantes, necesidades, expectativas de los alumnos y que quieren traer a la clase como parte de la tarea reflexiva. Aquí se intentaría conjugar las propias aspiraciones del docente con la de los alumnos. Pero esto es algo que debemos construir. Langón habla de un ethos común mínimo a construir como una posibilidad y de un logos común que se logra a través de los diversos pequeños logos o diálogos. Éstos mínimos se hacen necesarios para lograr un clima de trabajo favorable.

Pensar un curso como obra tiene mucho de creatividad y placer pero también de trabajo y esfuerzo. El profesor es sin duda el que propone, pero son varias las condiciones que hacen disponer el trabajo: los alumnos, los tiempos educativos, los procesos personales y grupales, los filósofos en cuestión, etc. Esta tarea tiene que ser realizada en toda su amplitud con gran responsabilidad. En palabras de Raffin: «En la medida en que el profesor construye su curso, efectúa un verdadero trabajo de elaboración de una problemática de articulación de conceptos que son plenamente suyos» ${ }^{24}$. El esfuerzo por pensar el curso lo hace al profesor exponer con ello también su propio pensamiento y ello es parte de la tarea intelectual que debemos desarrollar.

Es en este juego donde tomamos algo de la experiencia de los alumnos y los hacemos jugar con las inquietudes propias, centrándose en la necesidad que demande el curso en cuestión, no debemos perder de vista la necesidad de un trabajo pensado y ordenado. Por ello debe pensarse cada paso en función del edificio estructural que será el curso. Dice Derrida en la sexta exigencia: «...la unidad, e incluso la arquitectura de la disciplina requieren cierta reunión organizada de esta duración. Es necesario evitar la presentación desordenada... $\rangle^{25}$. Los problemas a trabajar y las preguntas que van surgiendo de ellos

\footnotetext{
22. Raffin, «Nuestra concepción de la...».

23. Fullat, «El pasmo de...», 53.

24. Raffin, «Nuestra concepción de la...».

25. Derrida, «Las antinomias de la disciplina filosófica...».
} 
pueden ir de manera enlazada de forma que organice el discurso y haga que los conceptos vayan asentándose poco a poco, dando consistencia a un posterior razonamiento.

\section{Sexto: la filosofía como relación de ausencias}

Muchas veces experimento en el diálogo con los alumnos que se hace presente algo que no está explícito, pero que va apareciendo y de alguna manera es invocado, llamado al aula de clase. Se da en esos momentos un encuentro entre ausencias que están presentes en los alumnos y en mí mismo como docente. Pueden ser ausencias de saberes, de ignorancias o de preguntas, que van acompañándonos. Incluso ausencia de lo que estamos buscando. Esta ausencia se puede captar en la carencia que experimentamos en el esfuerzo reflexivo y a su vez en esa necesidad de alcanzar llenar ese hueco existencial. Dicho de mejor manera: «...la filosofía es al mismo tiempo deseo de saber y carencia de saber. No sería posible enseñarla y aprenderla sin habilitar la posibilidad de ese deseo y el reconocimiento de esa carencia... $\nu^{26}$.

Por lo tanto, filosofar implica experimentar esa tensión entre lo que no se posee (en el mejor o más fuerte significado de poseer, digamos, de lo que uno se apropia por derecho) pero que aun sin estar presente en nosotros, nos atrae hacia sí. Esa experiencia se puede vivir en la clase de filosofía, entre los alumnos que buscan algo de lo que aun no pueden hablar pues aun no lo conocen. Dice Heidegger: «...lo que ha de pensarse le vuelve las espaldas al hombre. Se le sustrae. Mas ¿cómo podemos saber lo más mínimo y ni aun nombrar aquello que se nos sustrae desde siempre? Lo que se sustrae deniega su advenimiento.... ${ }^{27}$. Es que en filosofía la actitud filosófica se va desarrollando a medida que nos damos cuenta de que lo que hablamos tiene que ver con nosotros, donde reconocemos algo que nos es propio pero que no estaba en nosotros, pero desde que lo pensamos y lo nombramos ya deja de ser ajeno.

Por eso en filosofía es necesario lograr desarrollar las competencias que ya poseemos, o sea, facultades, habilidades, deseos, etc. Langón habla de redescubrir los sentidos para aprender captar realidades a las cuales no estamos habituados y que han adormecido a nuestros sentidos (cercana a la inteligencia sentiente de Zubiri). Si la filosofía apasiona debe ser experimentada mucho más allá de lo intelectual, debe llevarnos al estremecimiento, a poder percibir sentimientos realmente fuertes. ¿Quién puede leer a Sartre sin experimentar la "náusea"? ¿Quién puede leer a Dussel y experimentar la injusticia, y verla en nuestro alrededor? ¿Quién lee a Freire y no siente indignación ante los problemas que causa el capitalismo a la educación actual? En estas relaciones de ausencias se puede dar el encuentro de lo inesperado, de lo que no se puede planificar en un curso de filosofía.

\section{Séptimo: la filosofía creadora de espacios de reflexión}

Normalmente se considera a la filosofía a nivel formal, dentro de ciertos espacios institucionales o académicos, con planificaciones prediseñadas como proceso necesario y

26. Isabel Gonzáles, Janet Tourn y Mónica Planchón, «Ideas para una didáctica filosófica en la formación docente», Revista Convocación, n. ${ }^{\circ} 6$ (marzo, 2012): 23.

27. Martin Heidegger, ¿Qué significa pensar? (La Plata:Terramar, 2005): 17. 
casi obligatorio, de manera sistematizada. Pero no es este el único lugar en el cual la misma filosofía se desarrolla. Visto así podría ser un intento de aplicar cierto «control» a los espacios reflexivos de los alumnos limitando no sólo su libertad sino ahogando posibles construcciones de nuevos espacios. Pero sin duda no son los únicos. Cuando filosofamos lo hacemos desde lo que somos y hacemos, en cualquier lugar en donde estemos y desde las experiencias de vida que van marcando nuestra historia.

Las experiencias de la vida influyen en nuestro pensar y sin duda que hacemos filosofía desde lo que somos con-otros, desde-otros y hacia-otros, por eso no podemos saltearnos los espacios que ya se han generado en nuestra época como oportunidad, encuentros y momentos de reflexión filosófica. Dice Langón: «Filosofar es una actividad concreta que se hace siempre en ámbitos más precisos y determinados...en un aula...en un café filosófico...en una comunidad de indagación...en un taller...en un grupo de amigos...en una asamblea... ${ }^{28}$. Y aunque esto lo haya dicho en el año 2000 creo que aun siguen faltando estos espacios en nuestras instituciones educativas. Espacios de verdadero debate filosófico que pueden tomar como escusa cualquier tema o problema, pero que nos lleve al trabajo filosófico por el necesario gusto de hacerlo.

Si bien creo que se están dando pasos, con este fin de abrir a todos el encuentro filosófico (congresos, encuentros, coloquios, entre otros), aun falta algo que sea, de alguna manera, más espontáneo y más desde la cotidianeidad propia del estudiante. Aun nos falta generar estos espacios reflexivos que la misma filosofía propone. Muchas veces sentí el miedo de atravesar los años de formación docente sin experimentar un verdadero encuentro con otros, de intercambio filosófico, de cruces de reflexiones, de esfuerzo conjunto para resolver problemas que tienen que ver con alumnos y profesores. Uno de los grandes peligros sigue siendo el de caer en la individualidad y egocentrismo intelectual, que a menudo vemos en los pasillos de nuestras instituciones formadoras o de centros de estudios terciarios donde la Academia predomina y seduce.

Por eso debemos aprovechar instancias como las anteriores, en las cuales podemos reflexionar sobre la filosofía, su enseñanza, su aprendizaje, sus ventajas y sus deficiencias, sus posibilidades y obstáculos, ya que junto al tiempo de formación se va uniendo el de la práctica. Y está demás decir lo difícil que se nos hace encontrar un alumnado dispuesto a filosofar. Por ello el espacio de compartir e intercambiar los diversos procesos es necesario para poder sobrellevar juntos las contrariedades que a nivel general atraviesa la educación en la mayoría de los países latinoamericanos, que hacen aun más compleja la tarea de la filosofía en una época donde pensar no está de moda pues no da réditos.

\section{Conclusiones}

La didáctica filosófica desde la experiencia en la práctica docente me hace percibir la necesidad de una profundización y una continua actualización de su enseñanza y de su aprendizaje. Por defender la didáctica filosófica como una disciplina específica, es que creemos que a nivel educativo pareciera seguirse hablando de filosofía sin atenderse a lo verdaderamente importante y que reclama nuestra atención. Quizá a esto se

28. Langón, «Filosofía de la enseñanza de la...». 
refiere Heidegger cuando dice: «...lo gravísimo de nuestra época es que todavía no pensamos.... ${ }^{29}$. Y esto lo tomo en el sentido de que no estamos pensando realmente en las dificultades que enfrentamos a la hora de la enseñanza filosófica. Estamos caminando hacia ello pero aun falta un trabajo más integrado desde la formación del profesorado hasta un acompañamiento de los docentes que están ya trabajando hace años. Las generaciones de alumnos cambian rápido y nos exigen una continua renovación de los métodos, encares, propuestas, si es que queremos hacer filosofía en al aula.

La realidad de nuestros alumnos es muy variada y compleja: por un lado, la falta de estudio por diversas razones (falta de ganas, falta de gusto, falta de tiempo o de fuerzas); por otro lado, las deficiencias que presentan como producto de una no muy buena enseñanza escolar. También el problema de los que deben trabajar y estudiar, lo cual muchas veces hace más difícil su permanencia en al estudio. Por un lado la aceleración propia de la edad que busca una dinamicidad mayor en la clase, por otro el cansancio y el aburrimiento como consecuencia de los horarios impuestos por el sistema. Por un lado efusividad de la adolescencia en la búsqueda de experiencias fuertes, por otro las experiencias dolorosas de la vida que dejan huellas en la afectividad y obstaculizan el pensamiento. No pretendo agotar exhaustivamente la realidad, sólo son una enumeración de algunas de ellas. Pero se puede percibir cuán difícil es la tarea del docente cuando quiere ayudar a sus alumnos a aprender a filosofar.

Todas estas complejidades que no están en ningún manual del profesor de filosofía, deben estar presentes en la agenda del docente en el momento de pensar el curso, adaptándose a cada grupo y respetando sus particularidades. Pero no olvidemos que de esto trata la filosofía de la enseñanza de la filosofía, de reflexionar acerca de la práctica concreta de la misma filosofía. Raffin afirma que «...la didáctica de la filosofía, tiene una singularidad propia ya que es, como toda didáctica, por esencia ligada a la disciplina, puesto que ella reflexiona sobre su constitución así como sobre las dificultades y obstáculos que encuentra su aprendizaje... ${ }^{30}$.Y esto es lo que estoy tratando de hacer.

No obstante, de alguna manera como dice Langón: «...el problema está planteado desde el sujeto que aprende y la realización y desarrollo de sus aptitudes activas; y no desde el objeto enseñado por quien lo conoce, ni por la capacidad de esta enseñanza de hacer aprender ese objeto.... $\rangle^{31}$. Esto me sugiere que a la hora del trabajo filosófico en clase también hay que tener en cuenta varios problemas que provienen de los alumnos, pero que van más allá de la tarea del profesor de filosofía. Hablo de la formación de los primeros años de la enseñanza secundaria en la cual aun no aparece en los programas la filosofía. Hablo de los problemas de comprensión lectora con los cuales muchos alumnos llegan al bachillerato. Hablo del desconocimiento que traen acerca de la filosofía. En fin, creo que las preocupaciones deben ir más allá de nuestras clases y deben apuntar a una reestructura de los planes educativos.

29. Martin Heidegger, ¿Qué significa ..., 15.

30. Raffin, «Nuestra concepción de la...».

31. Langón, «Filosofía de la enseñanza de la...». 
Aunque no podemos escapar del riesgo en el cual nos introduce la misma filosofía, que es seguir adaptándola tanto al sujeto, que hace correr el peligro de perder el rigor y el esfuerzo reflexivo que conlleva el trabajo filosófico. Con Raffin creemos que la filosofía es más un tema de problematización y discusión que de una doctrina, o de un saber que genera resultados medibles. Entre los tiempos acotados, la falta de lectura, los problemas por falta de herramientas cognoscitivas, por no haber aprendido a desarrollar un razonamiento mínimo que permita argumentar soportando las opiniones contrarias sin renunciar a las propias y sin aceptar la contraria rápidamente, creo que los problemas exceden a la filosofía. Pero aun así son problemas que atañen a la filosofía.

Para terminar y ser coherente con mi planteamiento me veo en la exigencia de compartir mi experiencia filosófica en sus inicios. Como alumno de secundaria (realizada un poco más tarde de lo normal), aun tengo muy presente a quien fuera una de mis profesoras inspiradoras en mi vocación a la docencia de filosofía. Cursando el tercer año del Bachillerato, recuerdo que la mayoría de mis compañeros no lograban comprenderla cuando dictaba sus clases. Hablaba prolongadamente en el tiempo y emitía palabras de forma muy rápida. Éramos todos mayores de edad. La mayoría ya trabajábamos y era un grupo que estaba decido a seguir estudiando. Pero yo aun no decidía qué camino seguir. Un día, con el libro de Platón en la mano, nos leía algunos pasajes de la República que ningún compañero lograba entender. Allí fue mi encuentro con la filosofía.

El recuerdo más fuerte de ese día lo puedo describir de la siguiente manera: la profesora, parada al lado de la ventana que daba a la calle -por la cual veíamos la hermosa plaza que da a la avenida principal de la ciudad- hablaba de la democracia propuesta en las palabras de Platón. Con voz firme y convencida, como si estuviera fuera de sí, llevaba adelante una lectura que me hacía desear quizá lo mismo que ella deseaba: un futuro más justo en el cual todos recibiéramos lo que merecemos. Con los ojos hacia el cielo, su mirada parecía trascender el momento que vivíamos. Por momentos me olvidaba que estaba en clase y me preguntaba: ¿De qué habla esta profesora? ¿Por qué se emociona tanto al leer a Platón? ¿Qué le produce este filósofo? No encontré respuestas, sólo evocación de una experiencia. El maestro me hacía conmover...me hacía apasionar por algo que quizá yo aun no conocía pero que me atraía desde su misma ausencia. Desde esta vivencia tan fuerte en la cual se mezclan lo racional y lo afectivo, las ideas y los sentimientos, es que creo sentí el llamado a una vida filosófica.

Esto mismo debemos buscar con nuestros alumnos: provocarles una conmoción interior que los lleve a cambiar el lugar que ocupan en el mundo para poder transformarlo. En ello se juega también nuestra vocación si es que verdaderamente sentimos la filosofía como savia por nuestras venas, como alimento primordial de cada día. Nuestros jóvenes están cansados de palabras vacías. Ojalá ellos encuentren en nuestras clases un despertar al amor y una necesidad de una filosofía que los golpee, que los sacuda, que los transforme, que los impulse. Para ellos necesitamos hablar un lenguaje conmovedor que transmita vida, y que en ese lenguaje aparezca el ser de la filosofía que -como dice Heidegger cuando habla del lenguaje como la casa del $\operatorname{ser}^{32}$ - nos supere y que les hable mucho más allá de nuestras palabras y acciones. El lenguaje filosófico nos hace instrumentos necesarios para su actualización.

32. Cfr. Martin Heidegger, Carta sobre el humanismo (Madrid: Alianza, 2006). 


\section{ABSTRACT}

\section{Between the not knowing from who teaches and the not knowing from who learns: A Reflection from the Philosophical Practice}

This work reports on the meaning of philosophy for the author in their teaching path, and the perceptions along the years of their professional training. The educational process includes learning which implies the manipulation of certain knowledge as well as its practice. The author reflects on philosophy itself and its didactics, to inform that philosophy implies a didactic on its own. This process exposes difficulties with this subject with high school students, since the dynamic of the classroom reveals a complexity that enriches the philosophical thinking itself. The intellectual responsibility as educators, casts doubts on the philosophical vocation of those who take the challenge, among other aspects. That is why, even though a reflection from the experience of the author as an educator is presented, this article is a valid input to reason on the necessity of didactics in philosophy.

Key words: Philosophy, didactics, theoretical knowledge, practice and wisdom.

\section{RÉSUMÉ}

Entre la méconnaissance de celui qui enseigne et la méconnaissance de celui qui apprend: Une réflexion dès la pratique philosophique

Ce travail rend compte de ce qui a signifié la philosophie pour l'auteur dans son voyage à travers l'enseignement, et ses perceptions au cours des années de sa formation professionnelle. Le processus éducatif inclut un apprentissage qui implique la gestion de certains savoirs. L'auteur réfléchit sur la philosophie même et sa didactique afin d'informer qu'elle entraîne une didactique propre dans laquelle on découvre les difficultés posées dans le cours de l'enseignement secondaire, quand la dynamique de la salle de classe met en évidence une complexité qui enrichit la pensée philosophique. Le niveau de responsabilité intellectuelle en tant qu'enseignants remet en question la vocation philosophique de celui qui relève le défi, parmi autres aspects. Si bien il s'agit d'une réflexion de l'expérience de l'auteur comme enseignant, cet article est un apport clé qui invite à penser à la nécessité de constituer une didactique philosophique.

Mots clés: philosophie, didactique, savoir théorique, pratique, sagesse.

\section{Bibliografía}

Boavida, João. «De una didáctica de la filosofía a una filosofía de la educación». Revista Española de Pedagogía 64, n. 234 (mayo-agosto 2006): 205-225. https://www.jstor.org/stable/23766060?seq=1\#page_scan_ tab_contents

Beltrand Russel. Los problemas de la filosofia, http://www.enxarxa.com/biblioteca/RUSSELL\%20Los\%20problemas $\% 20 \mathrm{de} \% 201 \mathrm{a} \% 20$ filosofia.pdf

Berttolini, Marisa, Mauricio Langón e Isabel Quintela. Materiales para la construcción de cursos de filosofía: ¿Qué es filosofía?. Montevideo: A-Z, 1997.

Deleuze Gilles y Félix Guattari. ¿Qué es la filosofía?. Barcelona: Anagrama, 2005.

Derrida, Jacques. «Las antinomias de la disciplina filosófica». Carta enviada a los participantes del Colloque Rencontres Ècole et Philosophie, realizado en la Universidad de París X, Nanterre, 20 y 21 de octubre de 1984, en De droit à la philosophie 1990.

Fournet-Betacourt, Raúl. Transformación intercultural de la filosofía. Bilbao: Desclée de Brouwer, 2001. 
Fullat, Octavi. El pasmo de ser hombre. Barcelona: Ariel, 1994.

González, Isabel, Janet Tourn y Mónica Planchón. «Ideas para una didáctica filosófica en la formación docente». Revista Convocación, n. $^{\circ} 6$ (marzo, 2012): 22-25.

Heidegger, Martin. ¿Qué significa pensar? La Plata: Terrajar, 2005.

Carta sobre el humanismo. Madrid: Alianza, 2006.

Kusch, Rodolfo. Geocultura del hombre americano. Obras Completas, Tomo III. Córdoba: Fundación Ross, 1976.

Langón, Mauricio. «Filosofía de la enseñanza de la filosofía». Comunicación al Congreso Brasileiro de Professores de Filosofía. Piracicaba, noviembre, 2000.

«Adolescencias de la filosofía». Comunicación a las I Jornadas Internacionales de Filosofía, Infancias en la filosofía: experimentar el pensar, pensar la experiencia. Buenos Aires, Novedades Educativas, 22-23 de julio de 2005.

Pesce, Fernando. «La didáctica en la formación de docentes para la enseñanza media en Uruguay». Revista Intercambios 1, n. ${ }^{\circ} 1$ (2014): 56-61. http://intercambios.cse.edu.uy/la-didactica-en-la-formacion-de -docentes-para-la-ensenanza-media-en-uruguay/

Puchet, Enrique. De filosofía y educación. Cuidado de sí y conocimiento de sí. Montevideo: De la Fuga, 2013.

Raffin, Francoise. «Nuestra concepción de la didáctica». (Trad. Langón. M.), Revista Diotime L'Agora, n. ${ }^{\circ} 8$ (Francia, 2000).

Salazar-Bondi, Augusto. Didáctica de la filosofía. Lima: Fondo Editorial, 1995.

Sambarino, Mario. «La función sociocultural de la filosofía en América Latina». Primer Coloquio Nacional de Filosofía de México: Morelia-Michocán, 1975. La Filosofía actual en América Latina. Grijalbo, México (1976): 165-181. 